\title{
Non-Abelianizable First Class Constraints
}

\author{
Farhang Loran* \\ Department of Physics, Isfahan University of Technology (IUT) \\ Isfahan, Iran
}

\begin{abstract}
We study the necessary and sufficient conditions on Abelianizable first class constraints. The necessary condition is derived from topological considerations on the structure of gauge group. The sufficient condition is obtained by applying the theorem of implicit function in calculus and studying the local structure of gauge orbits. Since the sufficient condition is necessary for existence of proper gauge fixing conditions, we conclude that in the case of a finite set of non-Abelianizable first class constraints, the Faddeev-Popov determinant is vanishing for any choice of subsidiary constraints. This result is explicitly examined for $\mathrm{SO}(3)$ gauge invariant model.
\end{abstract}

*e-mail: loran@cc.iut.ac.ir 


\section{Introduction}

A gauge theory, in general, possesses a set of first class constraints, $\phi_{i}, i=1, \cdots, N$, satisfying the algebra

$$
\left\{\phi_{i}, \phi_{j}\right\}=\sum_{k=1}^{N} U_{i j}{ }^{k} \phi_{k}, \quad i, j=1, \cdots, N,
$$

in which the structure functions $U_{i j}{ }^{k}$ 's are generally some functions of phase space coordinates. One of the most interesting questions in constraint systems is the possibility of converting a given set of first class constraints to an equivalent Abelian set. By definition, Abelian constraints commute with each other, i.e. their Poisson brackets with each other are vanishing identically. There are various motivations for examining such a possibility. For example, first class constraints are generators of gauge transformation: $\delta_{i}^{\phi} F(z)=\left\{F(z), \phi_{i}\right\}[1]$. Since $U_{i j}{ }^{k}$ are functions of phase space coordinates, the full generator of gauge transformation is a nontrivial combination of first class constraints [2]. This combination is the simplest if first class constraints are Abelian i.e. when the Poisson brackets of these constraints with each other vanish identically.

Abelianization of first class constraints can also result in two more important simplifications. First, following Dirac's arguments, quantization of a set of first class constraints satisfying the algebra (1), where $U_{i j}{ }^{k}$ 's are not $c$-numbers, requires a definite operator ordering [1]. That is because in Dirac quantization, physical states are defined as null eigenstates of the operators $\hat{\phi}_{i}$ 's,

$$
\left.\hat{\phi}_{i} \mid \text { phys }\right\rangle=0 \text {, }
$$

in which the operator $\hat{\phi}_{i}$ 's are defined corresponding to the constraints $\phi_{i}$ 's. Definition (2) and the algebra (1) are consistent if the operators $\hat{U}_{i j}{ }^{k}$ 's, defined corresponding to the structure functions $U_{i j}{ }^{k}$ 's, sit on the left of the operators $\hat{\phi}_{i}$ 's similar to Eq.(1). The existence of such an operator ordering is not evident generally. Apparently, when first class constraints are Abelian, no such operator ordering should be considered. Second, in BRST formalism, the algebra (1), in general, leads to a complicated expansion of the BRST charge in terms of the ghosts. When first class constraints are Abelian, the generator of BRST transformation can be recognized in the most simple way [3].

Different methods for Abelianization of first class constraints are studied. Example are, Abelianization via constraint resolution $[3,4,5]$ or via generalized canonical transformation for general non-Abelian constraints (that satisfy a closed algebra) [6]. In reference [7] the authors study Abelianization via Dirac's transformation. In this method, one assumes that linear combinations of non-Abelian first class constraints (satisfying a closed algebra) exist that converts the given set of non-Abelian constraints to an equivalent set of Abelian constraints. In this way the problem of Abelianization is led to that of solving a 
certain system of first order linear differential equations for the coefficients of these linear combinations. In [8], it is shown that mapping each first class constraint to the surface of the other constraints, results in Abelian first class constraints. In [9] it is shown that the maximal Abelian subset of second class constraints can be obtained in the same way.

The domain of validity and/or applicability of the above methods can be determined by studying the necessary conditions on Abelianizable first class constraints. Topology of gauge group at each point $p$ of the phase space, which is uniquely determined by the structure coefficients $U_{i j}{ }^{k}(p)$, provides the necessary tools for this purpose. In fact, if at some point $p$, a non-Abelian set of first class constraints can be made Abelian, the corresponding gauge group should be topologically equivalent to the Abelian gauge groups, i.e. the group of Euclidean translations.

In [4] a method for Abelianization of first class constraints is proposed, which is based on the theorem of implicit differentiation (or the theorem of implicit function) and gives a sufficient condition on Abelianizable first class constraints. According to that theorem, if at some point $p, d \phi$ is maximal (see section 3), one can in principle, solve the equations $\phi_{i}\left(z_{1}, \cdots, z_{N} ; z_{a}^{\prime}\right)=0, i=1, \cdots, N$ as $z_{i}=z_{i}\left(z_{a}^{\prime}\right), i=1, \cdots, N$. It is shown in [4] that the constraints $\psi_{i}=z_{i}-z_{i}\left(z_{a}^{\prime}\right)$, are Abelian. Therefore, maximality is the sufficient condition for constraints to be Abelianizable. Using these results, we conclude that $U_{i j}{ }^{k}(p)$ 's determine whether the maximality condition is satisfied at $p$ or not.

Violation of maximality causes serious problems. For example, the norm of the constraint surface is not well defined in the neighborhood of maximality-violated regions. Furthermore, the necessary condition on subsidiary constraints (gauge fixing conditions) $\omega_{i}$ 's, i.e. $\operatorname{det}\left(\left\{\phi_{i}, \omega_{j}\right\}\right)_{p} \neq 0$, can not be satisfied if maximality is violated at $p$. This means that Faddeev-Popov determinant is vanishing regardless our choice of subsidiary constraints. The equivalence of Lagrangian and Hamiltonian formalism for constraint systems is also proven under the assumption that primary constraints satisfy the maximality condition all over the phase space [10].

Of course it should be noted that here we study only constraint systems with a finite set of first class constraints and it is not straightforward to generalize the results of this paper to systems with an infinite set of constraints, e.g. the SU(N) Yang-Mills theory (See appendix 2).

The organization of paper is as follows. In section 2, we study the topological conditions on Abelianizable first class constraints. In section 3, we discuss the maximality condition as the sufficient condition in two instructive ways: in subsection 3.1 by reviewing the Abelianization method of ref.[4] and in subsection 3.2 by studying the local structure of gauge orbits. In section 4, we examine our results explicitly by considering two simple examples. Section 5, is devoted to summary and conclusion. There are also two short 
appendices. Appendix 1 is a review of the method of obtaining orthogonal bases of a given vector space. The second appendix is a review of the constraint algebra of $S U(N)$ Yang-Mills model.

\section{Topological Considerations, A Necessary Condi- tion}

Assume a finite set of irreducible first class constraints $\phi_{i}, i=1, \cdots, N$. By definition,

$$
\left\{\phi_{i}, \phi_{j}\right\}=U_{i j}{ }^{k} \phi_{k}
$$

where $U_{i j}{ }^{k}\left(z^{\mu}\right)$ are some functions of phase space coordinates $z^{\mu}$ 's. $\left\{\phi_{i}, \phi_{j}\right\}$ stands for the Poisson bracket of $\phi_{i}$ and $\phi_{j}$ defined as follows:

$$
\left\{\phi_{i}, \phi_{j}\right\}=\frac{\partial \phi_{i}}{\partial z^{\mu}} J^{\mu \nu} \frac{\partial \phi_{j}}{\partial z^{\nu}}
$$

where $J^{\mu \nu}=\left\{z^{\mu}, z^{\nu}\right\}$ is a full rank antisymmetric tensor, e.g the symplectic two form,

$$
J=\left(\begin{array}{cc}
0 & 1 \\
-1 & 0
\end{array}\right)
$$

The gauge transformation of any function of phase space, $F(z)$ is given by $\delta_{i}^{\phi} F\left(z^{\mu}\right)=$ $\left.\left\{F, \phi_{i}\right\}\right|_{\Phi}$ where $\Phi$ is the constraint surface corresponding to the constraints $\phi_{i}=0$, $i=1, \cdots, N[1]$. Using Eq.(3), and the Jaccobi identity for Poisson brackets, one can show that for an arbitrary analytic function of phase space coordinates $F(z)$,

$$
\begin{aligned}
{\left[\delta_{i}^{\phi}, \delta_{j}^{\phi}\right] F(z) } & =\left.\left\{\left\{F(z), \phi_{j}\right\}, \phi_{i}\right\}\right|_{\Phi}-\left.\left\{\left\{F(z), \phi_{i}\right\}, \phi_{j}\right\}\right|_{\Phi} \\
& =\left.\left\{\left\{\phi_{i}, \phi_{j}\right\}, F(z)\right\}\right|_{\Phi} \\
& =\left.\left\{U_{i j}{ }^{k} \phi_{k}, F(z)\right\}\right|_{\Phi} \\
& =-\left.U_{i j}{ }^{k}\left\{F(z), \phi_{k}\right\}\right|_{\Phi} \\
& =-U_{i j}{ }^{k} \delta_{k}^{\phi} F(z) .
\end{aligned}
$$

Consequently, $\delta_{i}^{\phi}$ 's are elements of a Lie algebra with structure coefficients $-U_{i j}{ }^{k}$ 's:

$$
\left[\delta_{i}^{\phi}(p), \delta_{j}^{\phi}(p)\right]=-U_{i j}^{k}(p) \delta_{k}^{\phi}(p), \quad p \in \Phi,
$$

The corresponding Lie group is called the gauge group and the gauge orbits are the integral curves of $\delta_{i}^{\phi}(p)$ 's.

The main concept in our study is the concept of equivalence of two sets of constraints. Two sets of constraints are said to be equivalent at some point $p$ of the phase space if 1 ) 
the corresponding constraint surfaces are similar at some neighborhood of $p$ and 2) the resulting gauge transformations are equivalent $[4,11]$. These conditions are fulfilled using the following definition of equivalence:

Definition The set of constraints $\psi_{i}, i=1, \cdots, N$ are equivalent to $\phi_{i}$ 's, $i=1, \cdots, N$ at $p$ if 1) $p \in \Phi$ and $p \in \Psi, 2) T_{p} \Phi$ is homeomorphic to $T_{p} \Psi$ and 3) $G_{p}^{\phi}=G_{p}^{\psi}$.

$\Phi(\Psi)$ is the constraint surface corresponding to the constraints $\phi_{i}{ }^{\prime} \mathrm{s}\left(\psi_{i}\right.$ 's $)$ and $T_{p} \Phi\left(T_{p} \Psi\right)$ is the tangent space of $\Phi(\Psi)$ at $p . G_{p}^{\phi}$ and $G_{p}^{\psi}$ are the gauge groups generated by $\delta_{i}^{\phi}(p)$ 's and $\delta_{i}^{\psi}(p)$ 's respectively. Two topological spaces are said to be homeomorphic if there exist an invertible continuous map between them [12]. Since $\delta_{i}^{\phi}(p)$ 's $\left(\delta_{i}^{\psi}(p)\right)$ expand some subspace of the tangent space $T_{p} \Phi\left(T_{p} \Psi\right.$ ) (see subsection 3.2) and the equivalence of two Lie groups $G_{p}^{\phi}$ and $G_{p}^{\psi}$ requires that these subspaces be homeomorphic, ${ }^{1}$ the second and third conditions in the definition given above are consistent with each other.

If two topological spaces are homeomorphic, their topological invariants should be the same. It is important to note that this is a necessary and not a sufficient condition. Example of topological invariants are connectedness and compactness [12].

Abelianization of a set of non-Abelian first class constraints amounts to obtaining an equivalent set of Abelian constraints. The gauge group of Abelian constraints is homeomorphic to the group of Euclidean translations, i.e. $\mathcal{R}^{N}$, where $N$ is the number of first class constraints. $^{2}$ These are simply-connected and non-compact spaces. Consequently, the necessary (not sufficient) condition on constraints $\phi_{i}$ 's to be Abelianizable at some point $p$ is that the corresponding gauge group determined by $U_{i j}{ }^{k}(p)$ 's should be simplyconnected and non-compact. For example, if $U_{i j}{ }^{k}(p)$ are the structure coefficients of some compact group e.g. $S O(N)$, then the corresponding constraints can not be made Abelian at $p$. We call such sets of first class constraints, non-Abelianizable constraints.

As an example consider the $S O(3)$ gauge invariant model [13] where first class constraints are $L_{i}=\epsilon_{i j k} x_{j} p_{k}, i=1,2,3$ satisfying the algebra $\left\{L_{i}, L_{j}\right\}=\epsilon_{i j k} L_{k}$, in which $\epsilon_{i j k}$ is the Levi-Civita tensor. Consequently the gauge group is compact (homeomorphic to $\mathcal{S}^{2}$ ) and $L_{i}$ 's are non-Abelianizable.

\section{Maximality, A Sufficient Condition}

In this section we obtain the sufficient condition, which we call the maximality condition, on constraints to be Abelianizable. Obviously the sufficient condition is not satisfied in

\footnotetext{
${ }^{1}$ Equality, =, is a trivial homeomorphism given by the identity map

${ }^{2}$ Some directions of $\mathcal{R}^{N}$ can be compactified. A compactified direction corresponds to a $U(1)$ gauge symmetry. The simplest example of such systems is the Friedberg model [14]. Although we do not consider such cases here but generalization of the final result to these systems is straightforward.
} 
the case of non-Abelianizable constraints. As we will show this means that the FaddeevPopov determinant in such systems is vanishing for any choice of subsidiary constraints. In the following two subsections, we follow two different methods to study the maximality condition. The first one is based on the Abelianization via constraint resolution which is a well known method [4]. In the second method we study the local structure of gauge orbits. Although the following methods are basically equivalent but they clarify different aspects of the maximality condition.

\subsection{Resolution of Constraints}

Here we review the Abelianization method introduced in [4]. Assume a set of first class constraints $\phi_{i}, i=1, \cdots, N$ and the corresponding constraint surface $\Phi$. Consider a point $p \in \Phi$, where $d \phi$ is maximal. Maximality here means that there exist a subset of phase space coordinates, $z_{i}, i=1, \cdots, N$, such that

$$
\operatorname{det}\left(\frac{\partial \phi_{i}}{\partial z_{j}}\right)_{p} \neq 0
$$

In this case according to the theorem of implicit differentiation (or theorem of implicit function), one can in principle, solve equations $\phi_{i}\left(z_{i} ; z_{a}^{\prime}\right)=0, i=1, \cdots, N$ to obtain $z_{i}=z_{i}\left(z_{a}^{\prime}\right), i=1, \cdots, N$. One can show that the set of constraints $\psi_{i}=z_{i}-z_{i}\left(z_{a}^{\prime}\right)$ which by construction are equivalent to $\phi_{i}$ 's, are Abelian. This can be verified noting that

$$
\left\{\psi_{i}, \psi_{j}\right\}=\left\{z_{i}, z_{j}\right\}-\left\{z_{i}, z_{j}\left(z_{a}^{\prime}\right)\right\}-\left\{z_{i}\left(z_{a}^{\prime}\right), z_{j}\right\}+\left\{z_{i}\left(z_{a}^{\prime}\right), z_{j}\left(z_{a}^{\prime}\right)\right\}
$$

is independent of $z_{i}$ 's because $\left\{z^{\mu}, z^{\nu}\right\}=0, \pm 1$ (see Eq.(5)). Since the left hand side of Eq.(9) vanishes on the constraint surface (where $z_{i}=z_{i}\left(z_{a}^{\prime}\right)$ ), one concludes that it vanishes identically and consequently $\psi_{i}$ 's are Abelian [4]. Using the chain rule of partial differentiation, one can determine explicitly the gradient of constraints $\psi_{i}$ 's in terms of the gradient of $\phi_{i}$ 's, though $\psi_{i}$ 's are implicitly known. This has two consequences. Firstly, one can explicitly verify the equivalence of gauge transformations generated by $\psi_{i}$ 's and $\phi_{i}$ 's. Secondly, it determines the homeomorphism mentioned in section 2 between the tangent spaces (or gauge groups).

The violation of maximality condition has various geometrical consequences. For example, at any point $p$ where maximality is not satisfied, the dimensionality and the norm of the constraint surface is not well-defined. Furthermore, the tangent space of the constraint surface at $p$ is not homeomorphic to the tangent space at the regular points (where maximality is satisfied), though tangent spaces at regular points are all homeomorphic to each other.

In maximality-violated regions, the condition $\operatorname{det}\left(\left\{\phi_{i}, \omega_{j}\right\}\right) \neq 0$ on the subsidiary constraints (gauge fixing conditions) $\omega_{i}$ 's, can not be satisfied for any choice of analytic 
functions $\omega_{i}$ 's, because

$$
\operatorname{det}\left(\left\{\phi_{i}, \omega_{j}\right\}\right)=\operatorname{det}\left(\frac{\partial \phi_{i}}{\partial z^{\mu}} J^{\mu \nu} \frac{\partial \omega_{j}}{\partial z^{\nu}}\right), \quad i, j=1, \cdots, N
$$

is vanishing if,

$$
\operatorname{rank}\left(\frac{\partial \phi_{i}}{\partial z^{\mu}}\right)<N
$$

In other words, if a set of constraints do not satisfy the maximality condition, the FaddeevPopov determinant (10) is vanishing for any choice of subsidiary constraints $\omega_{i}$ 's.

Although maximality is a sufficient condition for Abelianization of first class constraints, it is not a necessary condition. For example consider the constraints of the Friedberg model [14] $\phi_{1}=p_{z}$ and $\phi_{2}=x p_{y}-y p_{x}$. These constraints form a set of Abelian constraint though they do not satisfy the maximality condition at the origin.

To emphasize the topological considerations discussed in section 2, let us consider again the $\mathrm{SO}(3)$ gauge model. One can easily verify that the constraints $L_{i}$ 's do not satisfy the maximality condition at any point of phase space. But, since maximality is not a necessary condition, using mere this result, one can not conclude that $L_{i}$ are not Abelianizable.

\subsection{The Space of Gauge Orbits}

The method of resolution of constraints studied above, considers only the equivalence of constraints in the sense that they should define the same constraint surface. But it does not verify the equivalence of the corresponding gauge groups explicitly. In the following, focusing on the local structure of gauge orbits, we obtain a complete description of equivalence of constraints and the concept of Abelianizablity.

Consider a point $p$ on the constraint surface. The gauge transformation generated by the first class constraints $\phi_{i}, i=1, \cdots, N$ at $p$ can be given as follows,

$$
\delta_{i} F=X_{i}(F)=X_{i}^{\mu} \frac{\partial}{\partial z^{\mu}} F,
$$

where

$$
X_{i}^{\mu}=\left(\frac{\partial \phi_{i}}{\partial z^{\nu}} J^{\nu \mu}\right)_{p}
$$

The vectors $X_{i}$ 's span a subspace of the tangent space $T_{p} \Phi$ and determine the direction of gauge transformation on the constraint surface at $p$. It is obvious that the maximality condition,

$$
\operatorname{rank}\left(\frac{\partial \phi_{i}}{\partial z^{\mu}}\right)_{p}=N
$$

is the necessary condition to obtain exactly $N$ independent vector $X_{i}$ 's. For further use, note that definition (13) implies that $X_{i}^{\mu} J^{\mu \nu} X_{j}^{\nu}=\left.\left\{\phi_{i}, \phi_{j}\right\}\right|_{p}$ is vanishing identically. The 
gauge fixing conditions $\omega_{i}$ 's can be defined as functions which gradient are proportional to $X_{i}$ 's, i.e.

$$
\frac{\partial \omega_{i}}{\partial z^{\mu}}=f_{i} X_{i}^{\mu}, \quad(\text { no sum over } i)
$$

where $f_{i}$ is some function that can be determined by solving the condition,

$$
\frac{\partial\left(f_{i} X_{i}^{\mu}\right)}{\partial z^{\nu}}=\frac{\partial\left(f_{i} X_{i}^{\nu}\right)}{\partial z^{\mu}}
$$

which simply means that,

$$
\frac{\partial^{2} \omega_{i}}{\partial z^{\mu} \partial z^{\nu}}=\frac{\partial^{2} \omega_{i}}{\partial z^{\nu} \partial z^{\mu}}
$$

The gauge fixing conditions $\omega_{i}$ 's defined by Eq.(15) satisfy the following relations:

$$
\delta_{i} \omega_{j}=f_{i} X_{i} \cdot X_{j}
$$

where $X_{i} \cdot X_{j}$ denotes the inner product of two vectors $X_{i}$ and $X_{j}$ given by the relation $X_{i} \cdot X_{j}=X_{i}^{\mu} X_{j}^{\mu}$.

The space of gauge orbits passing through $p$ spanned by the vectors $X_{i}^{\mu}$ 's, can be equivalently spanned by a set of orthonormal vectors $\tilde{X}_{i},\left(\tilde{X}_{i} \cdot \tilde{X}_{j}=\delta_{i j}\right)$. In appendix 1 , we review a well known method to obtain $\tilde{X}_{i}$ 's in terms of $X_{i}$ 's. Eqs.(13) and (15) can be used to define a new set of constraints $\tilde{\phi}_{i}$ and gauge fixing conditions $\tilde{\omega}_{i}$ 's in terms of $\tilde{X}_{i}$ 's. By construction,

$$
\left\{\tilde{\phi}_{i}, \tilde{\omega}_{j}\right\}=g_{i} \delta_{i j}
$$

where $g_{i}$ is some function of phase space. Since we are studying the system in an arbitrary small neighborhood of $p$, the function $g_{i}$ can be estimated as a constant that can be absorbed in $\tilde{\omega}_{i}$. Thus Eq.(19) can be rewritten in a more interesting form $\left\{\tilde{\phi}_{i}, \tilde{\omega}_{j}\right\}=\delta_{i j}$. By construction, $\tilde{\phi}_{i}$ 's are first class constraints equivalent to $\phi_{i}$ 's. ${ }^{3}$ Now using the following theorem one can show that the set of constraints $\tilde{\phi}_{i}$ 's are Abelian.

Theorem. Given a set of first class constraints $\phi_{i}$, if there exist a set of gauge fixing conditions $\omega_{i}$ 's such that $\left\{\phi_{i}, \omega_{j}\right\}=\delta_{i j}$, then $\phi_{i}$ 's are Abelian.

Proof. Assume that the algebra of constraints is given by the relation $\left\{\phi_{i}, \phi_{j}\right\}=$ $U_{i j}{ }^{k} \phi_{k}$. Consider the constraints $\phi_{i}$ and $\phi_{j}$ and one of gauge fixing conditions, say $\omega_{k}$. Using Jaccobi identity, one can show that,

$$
\begin{aligned}
\left\{\omega_{k},\left\{\phi_{i}, \phi_{j}\right\}\right\} & =-\left\{\phi_{j},\left\{\omega_{k}, \phi_{i}\right\}\right\}-\left\{\phi_{i},\left\{\phi_{j}, \omega_{k}\right\}\right\} \\
& =\left\{\phi_{j}, \delta_{k i}\right\}-\left\{\phi_{i}, \delta_{j k}\right\}
\end{aligned}
$$

\footnotetext{
${ }^{3}$ From appendix 1 one verifies that $\tilde{X}_{i}=M_{i j} X_{j}$ where $M$ is some invertible matrix. Consequently one can show that firstly, $\tilde{X}_{i} J \tilde{X}_{j}$ is vanishing on the constraint surface which means that $\tilde{\phi}_{i}$ 's are first class and secondly, the corresponding gauge groups are equivalent
} 
which is vanishing identically. Therefore,

$$
\begin{aligned}
0 & =\left\{\omega_{k},\left\{\phi_{i}, \phi_{j}\right\}\right\} \\
& =\left\{\omega_{k}, U_{i j}{ }^{k^{\prime}} \phi_{k^{\prime}}\right\} \\
& =U_{i j}{ }^{k}-U_{i j}{ }^{k k^{\prime}} \phi_{k^{\prime}}
\end{aligned}
$$

where $U_{i j}{ }^{k k^{\prime}}=-\left\{\omega_{k}, U_{i j}{ }^{k^{\prime}}\right\}$. Consequently the algebra of constraints is given as follows,

$$
\left\{\phi_{i}, \phi_{j}\right\}=U_{i j}^{k k^{\prime}} \phi_{k} \phi_{k^{\prime}}
$$

By repeating the above calculation, arbitrary number of $\phi_{i}$ can appear on the right hand side of the above relation. To obtain a meaningful algebra, this chain of multiplying constraints should terminate somewhere which means that the right hand side of the above equation is vanishing identically. In other words, $\phi_{i}$ 's are Abelian.

Summarizing our results, if at some point $p$ on the surface of $N$ first class constraints, the maximality condition is satisfied then the space of gauge orbits at that point looks like $\mathcal{R}^{N}$ (spanned by $N$ orthogonal vectors $\tilde{X}_{i}$ 's). Furthermore there exist an equivalent set of Abelian constraints which can be recognized as generators of translation along orthogonal directions of $\mathcal{R}^{N}$. Consequently, if we are given a finite set of first class constraints, such that the corresponding space of gauge orbits looks like, say, a sphere, they satisfy the maximality condition nowhere on the constraint surface. Therefore for any choice of subsidiary constraints, the Faddeev-Popov determinant is vanishing. This result can also be verified noting that the Faddeev-Popov determinant is, in general, proportional to $\operatorname{det}\left(X_{i} \cdot X_{j}\right)_{N \times N}$ which can be non-vanishing only if maximality condition is satisfied and $X_{i}$ 's are exactly $N$ independent vectors (See Eq.(14)).

Finally let us briefly review the Abelianization method given in ref.[8]. There, using the Cauchy-Kowalevski theorem [15], it is concluded that for any analytic constraint $\phi$, the partial differential equation $\{\omega, \phi\}=1$ has at least one solution for $\omega$ that can be uniquely determined by the boundary conditions. $\omega$ is used to define a projection map to the constraint surface $\phi=0$. It is shown that after projecting the remaining constraints to the surface $\phi=0$, one finds a new set of constraints equivalent to the original set with an interesting property: the Poisson brackets of all mapped constraints with $\phi$ is vanishing identically. It is shown that using similar projection operators one can map all constraints to the surface of each other consistently, which results in an equivalent Abelian set of constraints. In addition it shown that one can obtain a set of subsidiary constraint such that $\left\{\phi_{i}, \omega_{j}\right\}=\delta_{i j}{ }^{4}$ Obviously, the domain of validity of this method is

\footnotetext{
${ }^{4}$ It is straightforward to show that, obtaining the projected constraints is equivalent to calculating the orthogonal vectors $\tilde{X}_{i}$ 's in terms of the original $X_{i}$ 's.
} 
determined by the domain of validity Cauchy-Kowalevski theorem. As one anticipates, this theorem is valid as far as the maximality condition is satisfied. See ref.[15] for details.

The result of this section can be used to prove the following theorem on second class constraints:

Theorem If a given set of second class constraints can be considered as the union of first class constraints and the corresponding gauge fixing conditions, then the subset of first class constraints is Abelianizable.

To prove this theorem note that by definition a set of second class constraints $\psi_{I}$ 's satisfy the relation $\left.\operatorname{det}\left(\left\{\psi_{I}, \psi_{J}\right\}\right)\right|_{\psi} \neq 0$. If $\psi_{I}$ 's are a combination of some first class constraints $\phi_{i}$ (namely $\left.\left\{\phi_{i}, \phi_{j}\right\}\right|_{\psi}=0$ ) and gauge fixing conditions $\omega_{i}$, the definition of second class constraints gives,

$$
\operatorname{det}\left(\begin{array}{cc}
0 & A \\
-A & \left\{\omega_{i}, \omega_{j}\right\}
\end{array}\right) \neq 0
$$

where $A_{i j}=\left\{\phi_{i}, \omega_{j}\right\}$. Thus $\operatorname{det} A \neq 0$ and the maximality condition is satisfied. For more details see ref.[9] and references therein.

\section{Examples}

In this section we consider the simplest examples of Abelianizable and non-Abelianizable constraints. The non-Abelianizable constraints that we study here are the non-Abelian constraints of $S O(3)$ gauge model. We explicitly show that the Faddeev-Popov determinant is vanishing for any choice of gauge fixing conditions.

1. Abelianizable Constraints. Consider a system given by the following constraints,

$$
\phi_{1}=p_{x}, \quad \phi_{2}=p_{y}-e^{x} p_{y}
$$

which satisfy the algebra, $\left\{\phi_{1}, \phi_{2}\right\}=e^{x} \phi_{1}$. Obviously, this set of constraints is equivalent to the Abelian set $\tilde{\phi}_{1}=p_{x}$ and $\tilde{\phi}_{2}=p_{y}$. It is interesting to obtain these Abelian constraints using the method of subsection 3.2. First note that

$$
X_{1}=\left(\begin{array}{l}
1 \\
0 \\
0 \\
0
\end{array}\right), \quad X_{2}=\left(\begin{array}{c}
-e^{x} \\
1 \\
0 \\
0
\end{array}\right)
$$


where, for example, $X_{1}=J .\left.\nabla \phi_{1}\right|_{\phi_{1}, \phi_{2}}$, in which

$$
\nabla \phi_{1}=\left(\begin{array}{c}
\frac{\partial}{\partial x} \\
\frac{\partial}{\partial y} \\
\frac{\partial}{\partial p_{x}} \\
\frac{\partial}{\partial p_{y}}
\end{array}\right) \phi_{1}
$$

Using the method of Appendix 1, one obtains,

$$
\tilde{X}_{1}=\left(\begin{array}{c}
1 \\
0 \\
0 \\
0
\end{array}\right), \quad \tilde{X}_{2}=\left(\begin{array}{l}
0 \\
1 \\
0 \\
0
\end{array}\right)
$$

and consequently

$$
\nabla \tilde{\phi}_{1}=-J . \tilde{X}_{1}=\left(\begin{array}{c}
0 \\
0 \\
1 \\
0
\end{array}\right), \quad \nabla \tilde{\phi}_{2}=-J . \tilde{X}_{2}=\left(\begin{array}{l}
0 \\
0 \\
0 \\
1
\end{array}\right),
$$

which gives, $\tilde{\phi}_{1}=p_{x}$ and $\tilde{\phi}_{2}=p_{y}$.

2. Non-Abelianizable Constraints. Consider the constraints of $S O(3)$ gauge model, $L_{i}=\epsilon_{i j k} x_{j} p_{k}, i=1,2,3$. Assume three arbitrary subsidiary constraints $\omega_{i}$ 's. Since $L_{i}$ 's are non-Abelianizable, the Faddeev-Popov determinant $\operatorname{det}\left(\left\{\omega_{i}, L_{j}\right\}\right)$ is vanishing as can be verified as follows. Using the equality,

$$
\operatorname{det}\left(\begin{array}{lll}
a_{11} & a_{12} & a_{13} \\
a_{21} & a_{22} & a_{23} \\
a_{31} & a_{32} & a_{33}
\end{array}\right)=\epsilon_{i j k} a_{1 i} a_{2 j} a_{3 k}
$$

one finds that

$$
\begin{aligned}
\operatorname{det}\left(\left\{\omega_{i}, L_{j}\right\}\right) & =\epsilon_{i j k}\left\{\omega_{1}, L_{i}\right\}\left\{\omega_{2}, L_{j}\right\}\left\{\omega_{3}, L_{k}\right\} \\
& =-\epsilon_{i j k} \epsilon_{i a_{1} b_{1}} \epsilon_{j a_{2} b_{2}} \epsilon_{k a_{3} b_{3}} \prod_{c=1}^{3}\left(\frac{\partial \omega_{c}}{\partial x_{a_{c}}} x_{b_{c}}+\frac{\partial \omega_{c}}{\partial p_{a_{c}}} p_{b_{c}}\right),
\end{aligned}
$$

Two generic terms in the above sum can be distinguished:

$$
P=\epsilon_{i j k} \epsilon_{i a a^{\prime}} \epsilon_{j b b^{\prime}} \epsilon_{k c c^{\prime}}\left(\frac{\partial \omega_{1}}{\partial x_{a}} x_{a^{\prime}}\right)\left(\frac{\partial \omega_{2}}{\partial x_{b}} x_{b^{\prime}}\right)\left(\frac{\partial \omega_{3}}{\partial x_{c}} x_{c^{\prime}}\right),
$$

and

$$
Q=\epsilon_{i j k} \epsilon_{i a a^{\prime}} \epsilon_{j b b^{\prime}} \epsilon_{k c c^{\prime}}\left(\frac{\partial \omega_{1}}{\partial x_{a}} x_{a^{\prime}}\right)\left(\frac{\partial \omega_{2}}{\partial x_{b}} x_{b^{\prime}}\right)\left(\frac{\partial \omega_{3}}{\partial p_{c}} p_{c^{\prime}}\right)
$$


To calculate $P$, one realizes three generic terms:

$$
\begin{aligned}
P_{1} & =\left(\frac{\partial \omega_{1}}{\partial x}\right)\left(\frac{\partial \omega_{2}}{\partial x}\right)\left(\frac{\partial \omega_{3}}{\partial x}\right) \epsilon_{i j k} \epsilon_{i 1 a} \epsilon_{j 1 b} \epsilon_{k 1 c}\left(x_{a} x_{b} x_{c}\right), \\
P_{2} & =\left(\frac{\partial \omega_{1}}{\partial x}\right)\left(\frac{\partial \omega_{2}}{\partial x}\right)\left(\frac{\partial \omega_{3}}{\partial y}\right) \epsilon_{i j k} \epsilon_{i 1 a} \epsilon_{j 1 b} \epsilon_{k 2 c}\left(x_{a} x_{b} x_{c}\right), \\
P_{3} & =\left(\frac{\partial \omega_{1}}{\partial x}\right)\left(\frac{\partial \omega_{2}}{\partial y}\right)\left(\frac{\partial \omega_{3}}{\partial z}\right) \epsilon_{i j k} \epsilon_{i 1 a} \epsilon_{j 2 b} \epsilon_{k 3 c}\left(x_{a} x_{b} x_{c}\right) .
\end{aligned}
$$

$P_{1}=0$ because here, $(i, j, k) \in\{2,3\}$ and consequently $\epsilon_{i j k}$ is vanishing. $P_{2}=0$ because $\epsilon_{i 1 a} \epsilon_{j 2_{b}} x_{a} x_{b}$ is symmetric under $a \leftrightarrow b$ and consequently under $i \leftrightarrow j$, though $\epsilon_{i j k}=-\epsilon_{j i k}$. In addition, $P_{3}=-y z x+z x y=0$ (The first term corresponds to $a=2$ and the second terms corresponds to $a=3$ in Eq.(33)).

$Q$ is the sum of four generic terms:

$$
\begin{aligned}
Q_{1} & =\frac{\partial \omega_{1}}{\partial x} \frac{\partial \omega_{2}}{\partial x} \frac{\partial \omega_{3}}{\partial p_{x}} \epsilon_{i j k} \epsilon_{i 1 a^{\prime}} \epsilon_{j 1 b^{\prime}} \epsilon_{k 1 c^{\prime}} x_{a^{\prime}} x_{b^{\prime}} p_{c^{\prime}}, \\
Q_{2} & =\frac{\partial \omega_{1}}{\partial x} \frac{\partial \omega_{2}}{\partial x} \frac{\partial \omega_{3}}{\partial p_{y}} \epsilon_{i j k} \epsilon_{i 1 a^{\prime}} \epsilon_{j 1 b^{\prime}} \epsilon_{k 2 c^{\prime}} x_{a^{\prime}} x_{b^{\prime}} p_{c^{\prime}} \\
Q_{3} & =\frac{\partial \omega_{1}}{\partial x} \frac{\partial \omega_{2}}{\partial y} \frac{\partial \omega_{3}}{\partial p_{x}} \epsilon_{i j k} \epsilon_{i 1 a^{\prime}} \epsilon_{j 2 b^{\prime}} \epsilon_{k 1 c^{\prime}} x_{a^{\prime}} x_{b^{\prime}} p_{c^{\prime}} \\
Q_{4} & =\frac{\partial \omega_{1}}{\partial x} \frac{\partial \omega_{2}}{\partial y} \frac{\partial \omega_{3}}{\partial p_{z}} \epsilon_{i j k} \epsilon_{i 1 a^{\prime}} \epsilon_{j 2 b^{\prime}} \epsilon_{k 3 c^{\prime}} x_{a^{\prime}} x_{b^{\prime}} p_{c^{\prime}}
\end{aligned}
$$

$Q_{1}$ and $Q_{2}$ are vanishing because under $i \leftrightarrow j, \epsilon_{i 1 a^{\prime}} \epsilon_{j 1 b^{\prime}} x_{a^{\prime}} x_{b^{\prime}}$ is symmetric but $\epsilon_{i j k}$ is antisymmetric. Using the identity, $\epsilon_{i j k} \epsilon_{j 2 b^{\prime}}=-\delta_{i 2} \delta_{k b^{\prime}}+\delta_{i b^{\prime}} \delta_{k 2}$, one can show that $Q_{3}$ and $Q_{4}$ are some combinations of $L_{i}$ 's. Therefore $Q$ is also vanishing on the constraint surface.

\section{Conclusion}

We found that first class constraints can be classified as Abelianizable and non-Abelianizable constraints. These classes are identified by topological invariants (e.g. compactness) of the corresponding gauge groups. The topology of a gauge group is uniquely determined by the structure coefficients of the gauge generators' algebra which are simply the structure functions of the constraint algebra calculated at some particular point of phase space. Since maximality is the necessary condition on a given set of first class constraints for existence of a proper set of gauge fixing conditions, i.e. a set of subsidiary constraints such that the Faddeev-Popov determinant is non-vanishing, we concluded that

1. These constraints are Abelianizable if there exist a set of subsidiary constraints such that Faddeev-Popov determinant is non-vanishing.

2. If these constraints are non-Abelianizable then Faddeev-Popov determinant is vanishing for any choice of gauge fixing conditions. 
We studied the $S O(3)$ gauge invariant model as an example.

Using the first result mentioned above, we found that if a set of second class constraints is considered as the union of first class constraints and the corresponding gauge fixing conditions, then the subset of first class constraints is Abelianizable.

\section{Appendix 1}

Here we briefly review the method of obtaining $N$ orthogonal vectors $\vec{u}_{i}$ in terms of a given set of $N$ linearly independent vectors $\vec{v}_{i}$. This is a well know method that can be found in elementary text books in mathematics. Assuming that $\vec{v}_{i} \cdot \vec{v}_{i}=1$, the set of orthogonal vectors $\vec{u}_{i}$ 's, up to some normalization constants can be obtained as follows:

$$
\begin{aligned}
\vec{u}_{1} & =\vec{v}_{1}, \\
\vec{u}_{2} & =\vec{v}_{2}-\left(\vec{u}_{1} \cdot \vec{v}_{2}\right) \vec{u}_{1}, \\
\vec{u}_{n+1} & =v_{n}-\sum_{i=1}^{n}\left(u_{i} \cdot \vec{v}_{n}\right) u_{i}, \quad n=2, \cdots, N .
\end{aligned}
$$

\section{Appendix 2}

In this appendix we study the algebra of $S U(N)$ Yang-Mills theory. It is well know that this model possesses an infinite set of non-Abelian constraints,

$$
\phi_{a}(\vec{x})=\partial_{i} \Pi_{i}^{a}-g f_{a b c} A_{i}^{b} \Pi_{i}^{c}
$$

where $f_{a b c}$ 's are the structure coefficients of $S U(N)$ algebra and $\Pi_{i}^{a}$ is the momentum field conjugate to gauge field $A_{i}^{a}$, i.e. $\left\{A_{i}^{a}(\vec{x}), \Pi_{j}^{b}(\vec{y})\right\}=\delta^{a b} \delta_{i j} \delta^{D}(\vec{x}-\vec{y})$. These constraints satisfy the following algebra,

$$
\left\{\phi_{a}^{{ }^{g}}, \phi_{b}{ }^{g_{2}}\right\}=g f_{a b c} \phi_{c}^{{ }^{g} g_{2}}
$$

in which $g_{1}(\vec{x})$ and $g_{2}(\vec{x})$ are some smooth functions and $\phi_{a}{ }^{g}=\int d^{3} x g(x) \phi_{a}(x)[3,4]$. Assuming $g_{i}=e^{-i p_{i} x}$ where the momentum space is a compactified lattice, which corresponds to lattice gauge theory on tori, we obtain a finite set of constraints satisfying the following closed algebra:

$$
\left\{\phi_{a}^{m}, \phi_{b}^{n}\right\}=g f_{a b c} \delta_{m+n-p} \phi_{c}^{p}
$$

$\phi_{a}^{m}$ 's are non-Abelianizable and consequently the corresponding Faddeev-Popov determinant is vanishing. It is important to examine any possible relation between this result and the appearance of Gribov copies in ordinary $S U(N)$ Yang-Mills theory. 


\section{Acknowledgement}

The financial support of Isfahan University of Technology (IUT) is acknowledged.

\section{References}

[1] P. A. M. Dirac, Can. J. Math. 2, (1950) 129 ; Proc. R. Soc. London Ser. A 246, (1958) 326; "Lectures on Quantum Mechanics" New York: Yeshiva University Press, 1964 ,

[2] C. Batlle, J. Gomis, X. Gracia and J. M. Pons, J. Math. Phys. 30 (6), (1989) 1345; J. M. Pons and J. A. Garcia, Int. J. Mod. Phys. A 15 (2000) 4681, hep-th/9908151.

[3] M. Henneaux, Phys. Rep. 126, (1985) 1;

[4] M. Henneaux and C. Teitelboim "Quantization of Gauge System" Princeton University Press, Princeton, New Jersey, 1992.

[5] J. Goldberg, E. T. Newman and C. Rovelli, J. Math. Phys. 32, (1991) 2739.

[6] P. G. Bergman and I. Goldberg, Phys. Rev. 98, (1955) 531; P. G. Bergman ibid. 98, (1955) 544.

[7] S. A. Gogilidze, A. M. Khvedelidze and V. N. Pervushin, J. Math. Phys. 37, (1996) 1760, hep-th/9504153.

[8] F. Loran, Phys. Lett. B547, (2002) 63, hep-th/0209180.

[9] F. Loran, Phys. Lett. B554, (2003) 207, hep-th/0212341.

[10] C. Battle, J. Gomis, J.M. Pons and N. Roman-Roy, J. Math. Phys. 27 (12) (1986) 2953.

[11] J. Govaerts, "Hamiltonian Quantisation and Constrained Dynamics", Leuven Notes in Theoretical and Mathematical Physics, Leuven University Press, 1991.

[12] J. R. Munkres, "Topology, A First Course", Prentice-Hall, Inc, Englewood Cliffs, New Jersey, 1975.

[13] J. Govaerts and J. R. Klauder, Annals Phys. 274 (1999), 251, hep-th/9809119.

[14] R. Friedberg, T. D Lee, Y. Pang and H. C. Ren, Ann. Phys. 246, (1996) 381.

[15] F. John, "Partial Differential Equations", vol. 1, Fourth Edition, Springer-Verlag, New York Inc., 1981. 